University of Wollongong

Research Online

Faculty of Engineering - Papers (Archive)

Faculty of Engineering and Information

Sciences

October 1993

\title{
Hartree-Fock method posed as a density-functional theory: Application to the Be atom
}

\author{
A. Holas \\ Polish Academy of Sciences, Poland \\ N. H. March \\ University of Oxford, UK \\ Y. Takahashi \\ University of Alberta, Canada \\ C. Zhang \\ University of Wollongong, czhang@uow.edu.au
}

Follow this and additional works at: https://ro.uow.edu.au/engpapers

Part of the Engineering Commons

https://ro.uow.edu.au/engpapers/281

\section{Recommended Citation}

Holas, A.; March, N. H.; Takahashi, Y.; and Zhang, C.: Hartree-Fock method posed as a density-functional theory: Application to the Be atom 1993.

https://ro.uow.edu.au/engpapers/281

Research Online is the open access institutional repository for the University of Wollongong. For further information contact the UOW Library: research-pubs@uow.edu.au 


\title{
Hartree-Fock method posed as a density-functional theory: Application to the Be atom
}

\author{
A. Holas \\ Institute of Physical Chemistry of the Polish Academy of Sciences, 44/52 Kasprzaka, 01-224 Warsaw, Poland \\ N. H. March \\ Theoretical Chemistry Department, University of Oxford, 5 South Parks Road, Oxford OX1 3UB, England \\ Y. Takahashi \\ Theoretical Physics Institute, University of Alberta, Edmonton, Canada T6G 2J1 \\ C. Zhang \\ Department of Physics, University of Wollongong, New South Wales 2500, Australia \\ and Theoretical Physics Institute, University of Alberta, Edmonton, Canada T6G $2 \mathrm{J1}$ \\ (Received 8 March 1993)
}

\begin{abstract}
The Hartree-Fock ground-state energy and electron density are first shown to be derivable from a local one-body effective potential $v(\mathbf{r})$. As a nontrivial example, attention is then focused on the Be atom and isoelectronic atomic ions, the wave functions being written in terms of the density amplitude and phase. Some related general comments on the two-level one-dimensional system are included; kineticenergy density is shown to be a local functional of electron density generated by the harmonic-oscillator potential.
\end{abstract}

PACS number(s): $31.10 .+\mathrm{z}, 31.90 .+\mathrm{s}, 71.10 .+\mathrm{x}$

\section{INTRODUCTION}

In early work, Stoddart and co-workers [1] reformulated Hartree-Fock theory in density-matrix language, via a local potential $V_{H}(\mathbf{r})$. However, this work was perturbative in character, the unperturbed density matrices being built from plane waves.

Therefore, in the current density-functional study, a much more general formulation of Hartree-Fock theory is presented in terms of a local potential $v_{\text {eff }}^{\mathrm{HF}}(\mathbf{r})$. The main application made of this somewhat formal theory, derived in Sec. II below, is to two-level problems. We have in mind specifically the $\mathrm{Be}$ atom with configuration $(1 s)^{2}(2 s)^{2}$, and its isoelectronic atomic ions; this two-level example is set out fully in Sec. III. Some further relevant comments are added on the density-functional theory of model two-level one-dimensional systems in a group of appendixes.

\section{CONSTRAINED-SEARCH FORMULATION OF THE HARTREE-FOCK APPROXIMATION}

We are dealing with an $\mathrm{N}$-electron system (atom, molecule, etc.) having, by assumption, a nondegenerate ground state (GS). The GS energy in the Hartree-Fock approximation (HFA) is given by

$$
E_{\mathrm{GS}}^{\mathrm{HF}}=\min _{\Psi_{D}}\left\langle\Psi_{D}\left|\hat{T}+\hat{V}_{e e}+\hat{V}_{e n}\right| \Psi_{D}\right\rangle,
$$

where $\Psi_{D}$ is a normalized $N$-electron Slater determinant, and $\widehat{T}, \widehat{V}_{e e}$, and $\widehat{V}_{e n}$ are $N$-electron operators for the kinetic, electron-electron repulsion, and electron-nuclear attraction energies.
Following Payne [2], who laid the foundation of the density-functional approach to the HFA, and Levy [3] (see also [4]), we can perform the minimization in Eq. (2.1) in two steps, namely

$E_{\mathrm{GS}}^{\mathrm{HF}}=\min _{\rho}\left\{\min _{\Psi_{D} \rightarrow \rho}\left\langle\Psi_{D}\left|\hat{T}+\hat{V}_{e e}+\hat{V}_{e n}\right| \Psi_{D}\right\rangle\right\}$,

where by $\Psi_{D} \rightarrow \rho$ we denote such $\Psi_{D}$ which integrates to the given electron density $\rho(\mathbf{r})$. Finally, Eq. (2.2) can be rewritten as

$$
E_{\mathrm{GS}}^{\mathrm{HF}}=E^{\mathrm{HF}}\left[\rho_{\mathrm{GS}}^{\mathrm{HF}}\right]=\min _{\rho}\left\{F^{\mathrm{HF}}[\rho]+V_{e n}[\rho]\right\}
$$

with the constraint

$$
\int d^{3} r \rho(\mathbf{r})=N
$$

and energy functionals

$$
V_{e n}[\rho]=\int d^{3} r v_{e n}(\mathbf{r}) \rho(\mathbf{r}),
$$

where $v_{e n}(\mathbf{r})$ is the external potential acting on an electron, and

$$
F^{\mathrm{HF}}[\rho]=\min _{\Psi_{D} \rightarrow \rho}\left\langle\Psi_{D}\left|\widehat{T}+\hat{V}_{e e}\right| \Psi_{D}\right\rangle
$$

In the exact theory, in the Hohenberg-Kohn formulation (see, e.g., [4]) the functional $F^{\mathrm{HF}}[\rho]$ is replaced by $F^{\mathrm{HK}}[\rho]$, defined similarly as in Eq. (2.5), but with $\Psi$ an arbitrary antisymmetric $N$-electron function in place of $\Psi_{D}$.

Denoting $\Psi_{D}^{\mathrm{min}} \rightarrow \rho$ the minimizing wave function in Eq. (2.5), we define 


$$
T^{\mathrm{HF}}[\rho]=\left\langle\Psi_{D}^{\min }|\widehat{T}| \Psi_{D}^{\min }\right\rangle
$$

as the kinetic-energy functional of HFA, and

$$
E_{x}^{\mathrm{HF}}[\rho]=\left\langle\Psi_{D}^{\min }\left|\hat{V}_{e e}\right| \Psi_{D}^{\min }\right\rangle-E_{e s}[\rho]
$$

as the exchange energy of HFA, where

$$
E_{e s}[\rho]=\frac{1}{2} \int d^{3} r_{1} d^{3} r_{2} \frac{\rho\left(\mathbf{r}_{1}\right) \rho\left(\mathbf{r}_{2}\right)}{\left|\mathbf{r}_{1}-\mathbf{r}_{2}\right|}
$$

is the classical (electrostatic) electron-electron repulsion energy. So the HF energy functional $E^{\mathrm{HF}}[\rho]$ present in Eq. (2.3a) can be rewritten in terms of conventional contributions (shown above to be functionals of $\rho$ ):

$E^{\mathrm{HF}}[\rho]=T^{\mathrm{HF}}[\rho]+V_{e n}[\rho]+E_{e s}[\rho]+E_{x}^{\mathrm{HF}}[\rho]$.

In analogy with the exact theory, we can also obtain the Slater-Kohn-Sham (SKS)-like version of HFA. For that reason we recall the definitions (see, e.g., [4]) of $T_{s}[\rho]$, the kinetic-energy functional of $N$ noninteracting electrons (again in constrained-search formulation)

$$
T_{s}[\rho]=\min _{\Phi_{D} \rightarrow \rho}\left\langle\Phi_{D}|\hat{T}| \Phi_{D}\right\rangle,
$$

and the corresponding exchange-energy functional

$$
E_{x}[\rho]=\left\langle\Phi_{D}^{\min }\left|\hat{V}_{e e}\right| \Phi_{D}^{\min }\right\rangle-E_{e s}[\rho]
$$

where $\Phi_{D}^{\min }$ is the Slater determinant which minimizes Eq. (2.10) and integrates to $\rho(\mathbf{r})$. In terms of these functionals we can define the correlation energy of HFA to be

$$
\begin{aligned}
E_{c}^{\mathrm{HF}}[\rho] & =F^{\mathrm{HF}}[\rho]-\left\{T_{s}[\rho]+E_{e s}[\rho]+E_{x}[\rho]\right\} \\
& =\left\{T^{\mathrm{HF}}[\rho]-T_{s}[\rho]\right\}+\left\{E_{x}^{\mathrm{HF}}[\rho]-E_{x}[\rho]\right\},
\end{aligned}
$$

and, therefore, the usual exchange-correlation energy of HFA as

$$
E_{x c}^{\mathrm{HF}}[\rho]=E_{x}[\rho]+E_{c}^{\mathrm{HF}}[\rho] .
$$

So Eq. (2.3a) can be rewritten as

$$
E_{\mathrm{GS}}^{\mathrm{HF}}=\min _{\rho}\left\{T_{s}[\rho]+V_{\mathrm{eff}}^{\mathrm{HF}}[\rho]\right\}
$$

where the effective-potential energy is

$$
V_{\mathrm{eff}}^{\mathrm{HF}}[\rho]=V_{e n}[\rho]+E_{e s}[\rho]+E_{x c}^{\mathrm{HF}}[\rho] .
$$

The Slater-Kohn-Sham-like equations of HFA for canonical orbitals, which follow from Eqs. (2.14) and (2.3b), are

$$
\begin{aligned}
& \left\{-\frac{1}{2} \nabla^{2}+v_{\mathrm{eff}}^{\mathrm{HF}}(\mathbf{r})\right\} \phi_{i}(\mathbf{r}, s)=\varepsilon_{i} \phi_{i}(\mathbf{r}, s) \quad i=1, \ldots, N, \\
& \sum_{s} \int d^{3} r\left|\phi_{i}(\mathbf{r}, s)\right|^{2}=1
\end{aligned}
$$

and where

$$
\begin{aligned}
v_{\mathrm{eff}}^{\mathrm{HF}}(\mathbf{r}) & =\delta V_{\mathrm{eff}}^{\mathrm{HF}}[\rho] / \delta \rho(\mathbf{r}) \\
& =v_{e n}(\mathbf{r})+v_{e s}(\mathbf{r})+v_{x c}^{\mathrm{HF}}(\mathbf{r}),
\end{aligned}
$$

$$
\begin{aligned}
& v_{e s}(\mathbf{r})=\int d^{3} r^{\prime} \rho\left(\mathbf{r}^{\prime}\right) /\left|\mathbf{r}-\mathbf{r}^{\prime}\right|, \\
& v_{x c}^{\mathrm{HF}}(\mathbf{r})=\delta E_{x c}^{\mathrm{HF}}[\rho] / \delta \rho(\mathbf{r}) .
\end{aligned}
$$

Equations (2.16) and (2.17) must be solved selfconsistently, because the electron density $\rho(\mathbf{r})$ occurring in Eqs. (2.17) - (2.19) is obtained as

$$
\rho_{\mathrm{HF}}(\mathbf{r})=\rho(\mathbf{r})=\sum_{i=1}^{N} \sum_{s}\left|\phi_{i}(\mathbf{r}, s)\right|^{2} .
$$

This demonstrates that the ground-state energy $E_{\mathrm{GS}}^{\mathrm{HF}}$ and density $\rho_{\mathrm{GS}}^{\mathrm{HF}}$ of HFA can be obtained not only from the traditional canonical Hartree-Fock equations with nonlocal potential (see, e.g., [4]), but also from the SKS-like equations (2.16) with a local effective potential $v_{\text {eff }}^{\mathrm{HF}}(\mathbf{r})$, Eq. (2.17). It should be mentioned that Eq. (4.16) of Payne [2], the analog of our Eq. (2.16a), is incomplete, because the contribution $\delta\left(T^{\mathrm{HF}}[\rho]-T_{s}[\rho]\right) / \delta \rho(\mathbf{r})$ to the effective potential is missed there.

In practice, the constrained search for $F^{\mathrm{HF}}[\rho]$, Eq. (2.5), can be performed by applying methods developed for such a search in the case of $T_{s}[\rho]$, Eq. (2.10) (see, e.g., [5] and [6]). The " $B$ " method of the last reference seems to be particularly suitable for systems with large $N$.

In Sec. III we will show how the scheme set out above works for a simple system.

\section{ANALYSIS OF FOUR-ELECTRON SYSTEM IN THE RESTRICTED HARTREE-FOCK APPROXIMATION}

The approach developed in Sec. II is applied now to a four-electron system having a singlet ground state (e.g., a $\mathrm{Be}$ atom and its isoelectronic atomic ions, or a hypothetical $\mathrm{He}_{2}$ molecule). Let the variational Slater determinant $\Psi_{D}$ be constructed of two spatial orbitals $\psi_{1}(\mathbf{r})$ and $\psi_{2}(\mathbf{r})$, doubly occupied, chosen to be real. They must be normalized in order to have $\Psi_{D}$ normalized. So $\Psi_{D}$ integrates to the following electron density:

$$
\rho(\mathbf{r})=2\left\{\psi_{1}^{2}(\mathbf{r})+\psi_{2}^{2}(\mathbf{r})\right\}
$$

In order to have satisfied $\Psi_{D} \rightarrow \rho$, we represent these two orbitals in terms of the given density $\rho(\mathbf{r})$ and the variational phase function $\theta(\mathbf{r})$ (see, e.g., [5]) as

$$
\begin{aligned}
& \psi_{1}(\mathbf{r})=[\rho(\mathbf{r}) / 2]^{1 / 2} \cos \theta(\mathbf{r}) \\
& \psi_{2}(\mathbf{r})=[\rho(\mathbf{r}) / 2]^{1 / 2} \sin \theta(\mathbf{r})
\end{aligned}
$$

So the functional $F^{\mathrm{HF}}[\rho]$, Eq. (2.5) [see also Eqs. (2.6) $-(2.8)]$ can be written as

$$
F^{\mathrm{HF}}[\rho]=\min _{\theta}\left\{\widetilde{T}[\rho, \theta]+E_{e s}[\rho]+\widetilde{E}_{x}[\rho, \theta]\right\},
$$

with the constraint

$$
\tilde{\mathcal{N}}[\rho, \theta] \equiv \int d^{3} r \rho(\mathbf{r}) \sin ^{2} \theta(\mathbf{r})=2,
$$


where (see [5])

$$
\begin{aligned}
\widetilde{T}[\rho, \theta] & =2 \int d^{3} r\left\{\frac{1}{2}\left|\nabla \psi_{1}(\mathbf{r})\right|^{2}+\frac{1}{2}\left|\nabla \psi_{2}(r)\right|^{2}\right\} \\
& =T_{W}[\rho]+\widetilde{E}_{P}[\rho, \theta]
\end{aligned}
$$

with

$$
T_{W}[\rho]=\frac{1}{2} \int d^{3} r\left|\nabla\left[\rho^{1 / 2}(\mathbf{r})\right]\right|^{2}
$$

as the Weizsäcker kinetic energy, and

$$
\widetilde{E}_{P}[\rho, \theta]=\frac{1}{2} \int d^{3} r \rho(\mathbf{r})|\nabla \theta(\mathbf{r})|^{2}
$$

as the Pauli energy, and (see, e.g., [4])

$$
\begin{aligned}
\widetilde{E}_{x}[\rho, \theta]=-\int & d^{3} r_{1} d^{3} r_{2}\left|\mathbf{r}_{1}-\mathbf{r}_{2}\right|^{-1} \\
& \times\left[\psi_{1}\left(\mathbf{r}_{1}\right) \psi_{1}\left(\mathbf{r}_{2}\right)+\psi_{2}\left(\mathbf{r}_{1}\right) \psi_{2}\left(\mathbf{r}_{2}\right)\right]^{2} \\
=-\frac{1}{4} \int & d^{3} r_{1} d^{3} r_{2}\left|\mathbf{r}_{1}-\mathbf{r}_{2}\right|^{-1} \rho\left(\mathbf{r}_{1}\right) \rho\left(\mathbf{r}_{2}\right) \\
& \times \cos ^{2}\left[\theta\left(\mathbf{r}_{1}\right)-\theta\left(\mathbf{r}_{2}\right)\right] .
\end{aligned}
$$

Constraint (3.3b) assures normalization of $\psi_{2}$, while the normalization of $\psi_{1}$ follows from the combination of constraints $(2.3 \mathrm{~b})$ and (3.3b).

The Euler-Lagrange equation corresponding to Eqs. (3.3) is

$$
\frac{\delta}{\delta \theta(\mathbf{r})}\left\{\widetilde{E}_{P}[\rho, \theta]+\widetilde{E}_{x}[\rho, \theta]-\lambda \widetilde{\mathcal{N}}[\rho, \theta]\right\}_{\rho}=0
$$

[variational partial differentiation at fixed $\rho(\mathbf{r})]$, i.e.,

$$
\nabla^{2} \theta(\mathbf{r})+\rho^{-1}(\mathbf{r})(\nabla \rho(\mathbf{r}))(\nabla \theta(\mathbf{r}))-\frac{1}{2} \int d^{3} r^{\prime}\left|\mathbf{r}-\mathbf{r}^{\prime}\right|^{-1} \rho\left(\mathbf{r}^{\prime}\right) \sin ^{2}\left[2 \theta(\mathbf{r})-2 \theta\left(\mathbf{r}^{\prime}\right)\right]+\lambda \sin (2 \theta(\mathbf{r}))=0 .
$$

The boundary conditions for $\theta(\mathbf{r})$ are discussed in Ref. [5] [they are the same as for functions $K_{i}(\mathbf{r})$ there]. Because the given $\rho(\mathbf{r})$ is present in Eqs. (3.9) and (3.3b), their solution $\{\lambda, \theta(\mathbf{r})\}$ is a functional of $\rho(\mathbf{r})$.

Now the ground-state density can be obtained from the Lagrange-Euler equation corresponding to Eqs. (2.3):

$\frac{\delta}{\delta \rho(\mathbf{r})}\left\{F^{\mathrm{HF}}[\rho]+V_{e n}[\rho]-\mu \int d^{3} r^{\prime} \rho\left(\mathbf{r}^{\prime}\right)\right\}=0$,

i.e.,

$$
\frac{\delta F^{\mathrm{HF}}[\rho]}{\delta \rho(\mathbf{r})}+v_{e n}(\mathbf{r})=\mu
$$

From Eqs. (3.3) - (3.7) we have

$$
\begin{aligned}
& \frac{\delta F^{\mathrm{HF}}[\rho]}{\delta \rho(\mathbf{r})}= \frac{\delta T_{W}[\rho]}{\delta \rho(\mathbf{r})}+\frac{1}{2}|\nabla \theta(\mathbf{r})|^{2}+v_{e s}(\mathbf{r}) \\
&-\frac{1}{2} \int d^{3} r^{\prime}\left|\mathbf{r}-\mathbf{r}^{\prime}\right|^{-1} \rho\left(\mathbf{r}^{\prime}\right) \\
& \quad \times \cos ^{2}\left[\theta(\mathbf{r})-\theta\left(\mathbf{r}^{\prime}\right)\right]+v_{\text {ind }}(\mathbf{r}),
\end{aligned}
$$

where the indirect contribution [via $\theta(\mathbf{r})]$ is $v_{\text {ind }}(\mathbf{r})=\int d^{3} r^{\prime} \frac{\delta \theta\left(\mathbf{r}^{\prime}\right)}{\delta \rho(\mathbf{r})} \frac{\delta}{\delta \theta\left(\mathbf{r}^{\prime}\right)}\left\{\widetilde{E}_{P}[\rho, \theta]+\widetilde{E}_{x}[\rho, \theta]\right\}_{\rho}$.

$$
\left\{-\frac{1}{2} \nabla^{2}+v_{e n}(\mathbf{r})+\frac{1}{2}|\nabla \theta(\mathbf{r})|^{2}+v_{e s}(\mathbf{r})-\frac{1}{2} \int d^{3} r^{\prime}\left|\mathbf{r}-\mathbf{r}^{\prime}\right|^{-1} \rho\left(\mathbf{r}^{\prime}\right) \cos ^{2}\left[\theta(\mathbf{r})-\theta\left(\mathbf{r}^{\prime}\right)\right]-\lambda \sin ^{2} \theta(\mathbf{r})\right\} \chi(\mathbf{r})=\mu \chi(\mathbf{r})
$$

for the density amplitude

$$
\chi(\mathbf{r})=\rho^{1 / 2}(\mathbf{r}) .
$$

This equation must be solved self-consistently, because all potential terms, except $v_{e n}(\mathbf{r})$, are functionals of $\rho$. The boundary conditions for $\chi(\mathbf{r})$ are the same as for a wave function, i.e., it must vanish at boundary. But its normal-
Using Eq. (3.8) this $v_{\text {ind }}$ can be rewritten as

$$
v_{\text {ind }}(\mathbf{r})=\int d^{3} r^{\prime} \frac{\delta \theta\left(\mathbf{r}^{\prime}\right)}{\delta \rho(\mathbf{r})} \lambda\left\{\frac{\delta \widetilde{N}[\rho, \theta]}{\delta \theta\left(\mathbf{r}^{\prime}\right)}\right\}_{\rho} .
$$

But differentiating Eq. (3.3b) we find

$$
\begin{aligned}
0 & =\frac{\delta}{\delta \rho(\mathbf{r})} \tilde{\mathcal{N}}[\rho, \theta[\rho]] \\
& =\sin ^{2} \theta(\mathbf{r})+\int d^{3} r^{\prime} \frac{\delta \theta\left(\mathbf{r}^{\prime}\right)}{\delta \rho(\mathbf{r})}\left\{\frac{\delta \tilde{\mathcal{N}}[\rho, \theta]}{\delta \theta\left(\mathbf{r}^{\prime}\right)}\right\}_{\rho} .
\end{aligned}
$$

Substituting result (3.15) into Eq. (3.14), we find finally

$$
v_{\text {ind }}(\mathbf{r})=-\lambda \sin ^{2} \theta(\mathbf{r})
$$

Recalling that (e.g., [5])

$$
\frac{\delta T_{W}[\rho]}{\delta \rho(\mathbf{r})}=-\frac{1}{2} \frac{\nabla^{2}\left[\rho^{1 / 2}(\mathbf{r})\right]}{\rho^{1 / 2}(\mathbf{r})},
$$

we rewrite Eq. (3.11) with (3.12) as a Schrödinger equation

ization, according to $(2.3 \mathrm{~b})$, is

$$
\int d^{3} r \chi^{2}(\mathbf{r})=4 \text {. }
$$

In Appendix A, it is shown how Eqs. (3.9) and (3.18) can be derived alternatively from the conventional HF equations. 
Analogical consideration can be performed for the SKS-like formulation of this HF problem, Eq. (2.14). Let the variational $\Phi_{D}$ of Eq. (2.10) be constructed [similarly to Eq. (3.2)] of two orbitals:

$$
\begin{aligned}
& \phi_{1}(\mathbf{r})=[\rho(\mathbf{r}) / 2]^{1 / 2} \cos \theta_{s}(\mathbf{r}), \\
& \phi_{2}(\mathbf{r})=[\rho(\mathbf{r}) / 2]^{1 / 2} \sin \theta_{s}(\mathbf{r}) .
\end{aligned}
$$

Then minimization (2.10) takes the form [compare Eqs. (3.3)-(3.6)]

$$
\begin{aligned}
& T_{s}[\rho]=T_{W}[\rho]+\min _{\theta_{s}} \widetilde{E}_{P}\left[\rho, \theta_{s}\right], \\
& \tilde{\mathcal{N}}\left[\rho, \theta_{s}\right]=2 .
\end{aligned}
$$

The corresponding Euler-Lagrange equation is

$$
\frac{\delta}{\delta \theta_{s}(\mathbf{r})}\left\{\widetilde{E}_{P}\left[\rho, \theta_{s}\right]-\lambda_{s} \widetilde{\mathcal{N}}\left[\rho, \theta_{s}\right]\right\}_{\rho}=0,
$$

i.e.,

$$
\nabla^{2} \theta_{s}(\mathbf{r})+\rho^{-1}(\mathbf{r})(\nabla \rho(\mathbf{r}))\left(\nabla \theta_{s}(\mathbf{r})\right)+\lambda_{s} \sin \left(2 \theta_{s}(\mathbf{r})\right)=0 .
$$

This equation was obtained earlier in [5] in a onedimensional version, and also in [6]. Although, at selfconsistency, the same density $\rho(\mathbf{r})=\rho_{\mathrm{GS}}^{\mathrm{HF}}(\mathbf{r})$ occurs both in Eqs. (3.24) and (3.9), nevertheless the eigenvalues and eigenfunctions of these equations, $\left\{\lambda_{s}, \theta_{s}(\mathbf{r})\right\}$ and $\{\lambda, \theta(\mathbf{r})\}$, respectively, must be different, because Eq. (3.9) differs from Eq. (3.24) by the term corresponding to exchange.

If, for $\rho=\rho_{\mathrm{GS}}^{\mathrm{HF}}$ taken from the conventional HF calculation, Eqs. (3.24) and (3.22b) are solved numerically, giving $\left\{\lambda_{s}\left[\rho_{\mathrm{GS}}^{\mathrm{HF}}\right], \theta_{s}\left[\rho_{\mathrm{GS}}^{\mathrm{HF}} ; \mathbf{r}\right]\right\}$, then the effective potential $v_{\mathrm{eff}}^{\mathrm{HF}}$, which generates this density $\rho_{\mathrm{GS}}^{\mathrm{HF}}$ can be obtained in terms of $\theta_{s}$ (see, e.g., [5])

$$
v_{\mathrm{eff}}^{\mathrm{HF}}(\mathbf{r})=\frac{1}{2} \frac{\nabla^{2} \phi_{1}(\mathbf{r})}{\phi_{1}(\mathbf{r})}=\frac{1}{2} \frac{\nabla^{2}\left\{(\rho(\mathbf{r}) / 2)^{1 / 2} \cos \theta_{s}(\mathbf{r})\right\}}{(\rho(\mathbf{r}) / 2)^{1 / 2} \cos \theta_{s}(\mathbf{r})},
$$

After subtracting from this the known $v_{e n}(\mathbf{r})$ and $v_{e s}(\mathbf{r})$ [see Eqs. (2.17) and (2.18)], the exchange-correlation potential of HFA $v_{x c}^{\mathrm{HF}}(\mathbf{r})$, is determined. It should be noted that all potentials are obtained with accuracy to an additive constant, because variational $\rho(\mathbf{r})$ is constrained by Eq. (2.3b). Usually such a constant is fixed by the choice of vanishing potential at large distances.

The Schrödinger equation for the density amplitude $\chi(\mathbf{r})$, Eq. (3.19), obtained from Eqs. (2.14) and (2.3b), is (see [5])

$$
\begin{array}{r}
\left\{-\frac{1}{2} \nabla^{2}+\frac{1}{2}\left|\nabla \theta_{s}(\mathbf{r})\right|^{2}-\lambda_{s} \sin ^{2} \theta_{s}(\mathbf{r})+v_{\mathrm{eff}}^{\mathrm{HF}}(\mathbf{r})\right\} \chi(\mathbf{r}) \\
=\mu_{s} \chi(\mathbf{r}) .
\end{array}
$$

We can also write the noninteracting kinetic energy for the HF density [compare (3.22a)]

$$
T_{s}\left[\rho_{\mathrm{GS}}^{\mathrm{HF}}\right]=T_{W}\left[\rho_{\mathrm{GS}}^{\mathrm{HF}}\right]+\widetilde{E}_{P}\left[\rho_{\mathrm{GS}}^{\mathrm{HF}}, \theta_{s}\left[\rho_{\mathrm{GS}}^{\mathrm{HF}}\right]\right],
$$

and the exchange energy for the HF density, Eq. (2.11) [see Eq. (3.7), but with $\psi_{i}$ replaced by $\phi_{i}, \theta$ by $\theta_{s}$ ]:

$$
E_{x}\left[\rho_{\mathrm{GS}}^{\mathrm{HF}}\right]=\widetilde{E}_{x}\left[\rho_{\mathrm{GS}}^{\mathrm{HF}}, \theta_{s}\left[\rho_{\mathrm{GS}}^{\mathrm{HF}}\right]\right]
$$

If orbitals $\psi_{1}(\mathbf{r})$ and $\psi_{2}(\mathbf{r})$ are known from the conventional HF calculations, then, according to Eq. (3.2), the phase function can be found to be

$$
\theta(\mathbf{r})=\theta\left[\rho_{\mathrm{GS}}^{\mathrm{HF}} ; \mathbf{r}\right]=\arctan \left(\psi_{2}(\mathbf{r}) / \psi_{1}(\mathbf{r})\right) .
$$

From this and from the known $\rho=\rho_{\mathrm{GS}}^{\mathrm{HF}}(\mathbf{r})$, various quantities can be calculated:

The kinetic energy [Eqs. (3.4)-(3.6)],

$$
T^{\mathrm{HF}}=T_{W}\left[\rho_{\mathrm{GS}}^{\mathrm{HF}}\right]+\widetilde{E}_{P}\left[\rho_{\mathrm{GS}}^{\mathrm{HF}}, \theta\left[\rho_{\mathrm{GS}}^{\mathrm{HF}}\right]\right],
$$

the exchange energy [Eq. (3.7)],

$$
E_{x}^{\mathrm{HF}}=\widetilde{E}_{x}\left[\rho_{\mathrm{GS}}^{\mathrm{HF}}, \theta\left[\rho_{\mathrm{GS}}^{\mathrm{HF}}\right]\right],
$$

and separate contributions to the total potential of the Schrödinger equation (3.18) [the constant $\lambda$ should be determined from Eq. (3.9) by substituting there the known functions $\theta(\mathbf{r})$, Eq. (3.29), and $\rho(\mathbf{r})$, Eq. (3.1)]. The HF correlation energy Eq. (2.12) can be obtained from Eqs. (3.27), (3.28), (3.30), and (3.31):

$$
\begin{array}{r}
E_{c}^{\mathrm{HF}}[\rho]=\frac{1}{2} \int d^{3} r \rho(\mathbf{r})\left\{|\nabla \theta(\mathbf{r})|^{2}-\left|\nabla \theta_{s}(\mathbf{r})\right|^{2}\right\} \\
-\frac{1}{4} \int d^{3} r_{1} d^{3} r_{2}\left|\mathbf{r}_{1}-\mathbf{r}_{2}\right|^{-1} \rho\left(\mathbf{r}_{1}\right) \rho\left(\mathbf{r}_{2}\right) \\
\times\left\{\cos ^{2}\left[\theta\left(\mathbf{r}_{1}\right)-\theta\left(\mathbf{r}_{2}\right)\right]\right. \\
\left.\quad-\cos ^{2}\left[\theta_{s}\left(\mathbf{r}_{1}\right)-\theta_{s}\left(\mathbf{r}_{2}\right)\right]\right\} .
\end{array}
$$

Since Eqs. (3.18) and (3.26) determine the same $\chi(\mathbf{r})$, their potential must be the same (with an additive constant). So, comparing these potentials and using Eq. (2.17), we obtain

$$
\begin{aligned}
v_{x c}^{\mathrm{HF}}(r)= & -\frac{1}{2} \int d^{3} r^{\prime}\left|\mathbf{r}-\mathbf{r}^{\prime}\right|^{-1} \rho\left(\mathbf{r}^{\prime}\right) \cos ^{2}\left[\theta(\mathbf{r})-\theta\left(\mathbf{r}^{\prime}\right)\right] \\
& +\frac{1}{2}|\nabla \theta(\mathbf{r})|^{2}-\frac{1}{2}\left|\nabla \theta_{s}(\mathbf{r})\right|^{2}-\lambda \sin ^{2} \theta(\mathbf{r}) \\
& +\lambda_{s} \sin ^{2} \theta_{s}(\mathbf{r}) .
\end{aligned}
$$

However, the particular terms of expression (3.33) cannot be identified with contributions due to separate terms seen in Eq. (2.13) with (2.12), because the "indirect" term, Eq. (3.16), is connected with the sum of the kinetic and exchange contributions [see Eq. (3.13)].

\section{DISCUSSION AND CONCLUSIONS}

The problem of finding an algorithm to calculate the noninteracting kinetic energy $T_{s}$ as a functional of $\rho(\mathbf{r})$ [see definition (2.10)], and also its functional derivative $\delta T_{s}[\rho] / \delta \rho(\mathbf{r})$, was solved in general in earlier work ([5]; see also [6]). In Appendix B, the application of that method to one-dimensional two-level systems is set out, and contact made with the work of Shao and Stezowski [7]. The question these workers raise is whether the kinetic-energy density $t_{s}(x)$ can be obtained in closed form as a local function of particle density $\rho(x)$ and its derivatives. Appendix $\mathbf{C}$ demonstrates that the answer is positive for $\rho(x)$ generated by a harmonic-oscillator po- 
tential. However, the method applicable for general one-dimensional potentials, considered in Appendix B, must be used for generating $\delta T_{s} / \delta \rho$.

To return to the main thrust of the present work, the conclusion of Stoddart and co-workers [1] that HartreeFock theory can be rewritten in terms of a local potential, which was only proved in [1] for perturbation theory based on plane waves, is demonstrated quite generally in Sec. II using tools of the density-functional theory. Section III has been concerned with rewriting the canonical HF problem in terms of density amplitude $\{\rho(\mathbf{r})\}^{1 / 2}$ and phase $\theta(\mathbf{r})$. The phase equation for the $\mathrm{Be}$ atom is a generalization of the earlier work of Dawson and March [8] to embrace HF theory. A final point to be stressed is that, in the customary language of density-functional theory, HF theory already provides a starting approximation for $E_{\mathrm{xc}}$, even though, in quantum-chemical language, it includes only exchange and has zero correlation.

\section{ACKNOWLEDGMENTS}

One of us (N.H.M.) wishes to thank Professor F. C. Khanna and Professor A. Z. Capri of the Theoretical Physics Institute at the University of Alberta, Edmonton, for generous support during two visits. Part of this collaboration was thereby made possible. Part of the contributions of A.H. and N.H.M. were also facilitated greatly by their presence at Condensed Matter Workshops at ICTP, Trieste.

\section{APPENDIX A: EQUATIONS DETERMINING $\theta(\mathbf{r})$ AND $\rho(r)$ OBTAINED FROM THE CONVENTIONAL HF EQUATIONS}

The restricted Hartree-Fock equations for two canonical orbitals of the four-electron problem are (see, e.g., [4]; real orbital chosen)

$$
\begin{aligned}
& -\frac{1}{2} \nabla^{2} \psi_{1}(\mathbf{r})+\left[v_{e n}(\mathbf{r})+v_{e s}(\mathbf{r})\right] \psi_{1}(\mathbf{r}) \\
& -\int d^{3} r^{\prime} k\left(\mathbf{r}, \mathbf{r}^{\prime}\right) \psi_{1}\left(\mathbf{r}^{\prime}\right)=\varepsilon_{1} \psi_{1}(\mathbf{r}), \\
& -\frac{1}{2} \nabla^{2} \psi_{2}(\mathbf{r})+\left[v_{e n}(\mathbf{r})+v_{e s}(\mathbf{r})\right] \psi_{2}(\mathbf{r}) \\
& -\int d^{3} r^{\prime} k\left(\mathbf{r}, \mathbf{r}^{\prime}\right) \psi_{2}\left(\mathbf{r}^{\prime}\right)=\varepsilon_{2} \psi_{2}(\mathbf{r}),
\end{aligned}
$$

where

$$
\begin{aligned}
v_{e s}(\mathbf{r}) & =\int d^{3} r^{\prime}\left|\mathbf{r}-\mathbf{r}^{\prime}\right|^{-1} \rho(\mathbf{r}) \\
& =\int d^{3} r^{\prime}\left|\mathbf{r}-\mathbf{r}^{\prime}\right|^{-1} 2\left[\psi_{1}^{2}\left(\mathbf{r}^{\prime}\right)+\psi_{2}^{2}\left(\mathbf{r}^{\prime}\right)\right], \\
k\left(\mathbf{r}, \mathbf{r}^{\prime}\right) & =\left|\mathbf{r}-\mathbf{r}^{\prime}\right|^{-1}\left[\psi_{1}\left(\mathbf{r}^{\prime}\right) \psi_{1}(\mathbf{r})+\psi_{2}\left(\mathbf{r}^{\prime}\right) \psi_{2}(\mathbf{r})\right] .
\end{aligned}
$$

Let us subtract Eq. (A2) multiplied by $\psi_{1}(\mathbf{r})$ from Eq. (A1) multiplied by $\psi_{2}(\mathbf{r})$ :

$$
-\frac{1}{2}\left(\psi_{2} \nabla^{2} \psi_{1}-\psi_{1} \nabla^{2} \psi_{2}\right)-\int d^{3} r^{\prime} k\left(\mathbf{r}, \mathbf{r}^{\prime}\right)\left[\psi_{2}(\mathbf{r}) \psi_{1}\left(\mathbf{r}^{\prime}\right)-\psi_{1}(\mathbf{r}) \psi_{2}\left(\mathbf{r}^{\prime}\right)\right]=\varepsilon_{1} \psi_{2} \psi_{1}-\varepsilon_{2} \psi_{1} \psi_{2} .
$$

So

$$
\frac{1}{2} \nabla\left(\psi_{1} \nabla \psi_{2}-\psi_{2} \nabla \psi_{1}\right)-\int d^{3} r^{\prime}\left|\mathbf{r}-\mathbf{r}^{\prime}\right|^{-1}\left[\psi_{2}(\mathbf{r}) \psi_{1}\left(\mathbf{r}^{\prime}\right)-\psi_{1}(\mathbf{r}) \psi_{2}\left(\mathbf{r}^{\prime}\right)\right]\left[\psi_{1}\left(\mathbf{r}^{\prime}\right) \psi_{1}(\mathbf{r})+\psi_{2}\left(\mathbf{r}^{\prime}\right) \psi_{2}\left(\mathbf{r}^{\prime}\right)\right]+\left(\varepsilon_{2}-\varepsilon_{1}\right) \psi_{1} \psi_{2}=0 .
$$

Now we take $\psi_{1}$ and $\psi_{2}$ in the form of (3.2) and calculate combinations:

$$
\begin{aligned}
& \psi_{1} \psi_{2}=\frac{1}{4} \rho \sin 2 \theta \\
& \psi_{1} \nabla \psi_{2}-\psi_{2} \nabla \psi_{1}=\frac{1}{2} \rho \nabla \theta, \\
& \psi_{1}\left(\mathbf{r}^{\prime}\right) \psi_{2}(\mathbf{r})-\psi_{2}\left(\mathbf{r}^{\prime}\right) \psi_{1}(\mathbf{r})=\frac{1}{2}\left[\rho(\mathbf{r}) \rho\left(\mathbf{r}^{\prime}\right)\right]^{1 / 2} \sin \left[\theta(\mathbf{r})-\theta\left(\mathbf{r}^{\prime}\right)\right], \\
& \psi_{1}\left(\mathbf{r}^{\prime}\right) \psi_{1}(\mathbf{r})+\psi_{2}\left(\mathbf{r}^{\prime}\right) \psi_{2}(\mathbf{r})=\frac{1}{2}\left[\rho(\mathbf{r}) \rho\left(\mathbf{r}^{\prime}\right)\right]^{1 / 2} \cos \left[\theta(\mathbf{r})-\theta\left(\mathbf{r}^{\prime}\right)\right]
\end{aligned}
$$

After substituting Eqs. (A7)-(A10) into (A6), we obtain Eq. (3.9) exactly, with

$$
\lambda=\varepsilon_{2}-\varepsilon_{1},
$$

which gives the interpretation of the eigenvalue of Eq. (3.9) in terms of eigenvalues of HF Eqs. (A1) and (A2).

Next let us sum Eq. (A1) multiplied by $\psi_{1}(\mathbf{r})$ and Eq. (A2) multiplied by $\psi_{2}(\mathbf{r})$ :

$$
-\frac{1}{2}\left(\psi_{1} \nabla^{2} \psi_{1}+\psi_{2} \nabla^{2} \psi_{2}\right)+\left(v_{e n}+v_{e s}\right)\left(\psi_{1}^{2}+\psi_{2}^{2}\right)-\int d^{3} r^{\prime} k\left(\mathbf{r}, \mathbf{r}^{\prime}\right)\left[\psi_{1}(\mathbf{r}) \psi_{1}\left(\mathbf{r}^{\prime}\right)+\psi_{2}(\mathbf{r}) \psi_{2}\left(\mathbf{r}^{\prime}\right)\right]=\varepsilon_{1} \psi_{1}^{2}+\varepsilon_{2} \psi_{2}^{2} .
$$

Using (3.2) and (3.19) we calculate

$$
\begin{aligned}
& \psi_{1} \nabla^{2} \psi_{1}+\psi_{2} \nabla^{2} \psi_{2}=\frac{1}{2} \chi\left\{\nabla^{2} \chi-(\nabla \theta)^{2} \chi\right\} \\
& \psi_{1}^{2}+\psi_{2}^{2}=\frac{1}{2} \rho=\frac{1}{2} \chi^{2} \\
& \varepsilon_{1} \psi_{1}^{2}+\varepsilon_{2} \psi_{2}^{2}=\varepsilon_{1}\left(\psi_{1}^{2}+\psi_{2}^{2}\right)+\lambda \psi_{2}^{2} \\
& =\frac{1}{2} \chi^{2}\left\{\varepsilon_{1}+\lambda \sin ^{2} \theta\right\}
\end{aligned}
$$

Equation (A11) was used in obtaining Eq. (A15).

After substituting Eqs. (A13)-(A15) and (A10) into (A12) we obtain Eq. (3.18) exactly, with

$$
\mu=\varepsilon_{1} \text {. }
$$

So the eigenvalue of an eigenequation determining $\chi(\mathbf{r})$ is equal to the lowest HF eigenvalue $\varepsilon_{1}$. 


\section{APPENDIX B: ONE-DIMENSIONAL TWO-LEVEL PROBLEM OF NONINTERACTING ELECTRONS}

As shown in [5], the problem of finding an algorithm to calculate $T_{s}[\rho]$ and $\delta T_{s} / \delta \rho$ for dimensionality $D=1$ and number of levels (singly occupied) $N=2$ requires solution of one auxiliary eigenequation [compare Eq. (3.24) for $D=3]$ :

$$
\theta_{s}^{\prime \prime}+\left(\rho^{\prime} / \rho\right) \theta_{s}^{\prime}+\varepsilon \sin \left(2 \theta_{s}\right)=0
$$

where $\varepsilon$ is its eigenvalue, and $\theta_{s}(x)$ the corresponding eigenfunction subject to the boundary condition

$$
\theta_{s}^{\prime}(x) \rightarrow 0 \text { at } x \rightarrow \text { (boundary), }
$$

and to a number of constraints

$$
\begin{aligned}
& \int d x \rho \sin ^{2} \theta_{s}=1, \\
& -\pi / 2<\theta_{s}(x)<\pi / 2, \\
& \theta_{s}(x) \text { possesses one node only, } \\
& \varepsilon>0 .
\end{aligned}
$$

The solution $\left\{\varepsilon, \theta_{s}(x)\right\}$ is a functional of $\rho$, because this function is present in Eqs. (B1) and (B3).

The kinetic-energy density $t_{s}(x)$, defined in terms of orbitals to be

$$
t_{s}(x)=-\frac{1}{2}\left\{\phi_{1}(x) \phi_{1}^{\prime \prime}(x)+\phi_{2}(x) \phi_{2}^{\prime \prime}(x)\right\},
$$

can be written in terms of this solution as (we drop subscript $s$ from $\theta_{s}$ to simplify notation)

$$
t_{s}(x)=\frac{1}{8} \frac{\left(\rho^{\prime}\right)^{2}}{\rho}-\frac{1}{4} \rho^{\prime \prime}+\frac{1}{2}\left(\theta^{\prime}\right)^{2} \rho,
$$

so it is a functional of $\rho$. From it the functional derivative of the (total) kinetic energy

$$
T_{s}=\int d x t_{s}(x)
$$

is obtained as

$$
\begin{aligned}
\frac{\delta T_{s}[\rho]}{\delta \rho(x)}= & \frac{1}{8}\left[\frac{\rho^{\prime}}{\rho}\right]^{2}-\frac{1}{4} \frac{\rho^{\prime \prime}}{\rho}+\frac{1}{2}\left(\theta^{\prime}\right)^{2} \\
& +\frac{1}{2} \tan \theta\left[\theta^{\prime \prime}+\frac{\theta^{\prime} \rho^{\prime}}{\rho}\right) .
\end{aligned}
$$

While Eqs. (B1) and (B8) were already known and used (e.g., [7], [9], and [10]), the recognition of Eq. (B1) as an eigenvalue problem, constrained by conditions (B2) $-(\mathrm{B} 6)$, represents a new point of view formulated in [5]. The given density $\rho(x)$ is supposed to be noninteracting $v$ representable and integrable to $N=2$, although the generating potential may remain unknown.

What is interesting is that Eqs. (B1)-(B6) can be solved analytically in the case of densities generated by some simple potentials. An example of a Coulomb potential case is considered in [5], while here we demonstrate this for the harmonic-oscillator (HO) potential, preparing the discussion with Refs. [7], [9], and [10] in the Appendix C.
The only necessary input information $\rho(x)$ has the following form for the $\mathrm{HO}$ case

$$
\rho(x)=\rho_{\mathrm{GS}}^{\mathrm{HO}}(x)=\pi^{-1 / 2}\left(1+2 x^{2}\right) \exp \left(-x^{2}\right),
$$

so

$$
\rho^{\prime}(x) / \rho(x)=2 x\left(1-2 x^{2}\right) /\left(1+2 x^{2}\right) .
$$

The solution of Eqs. (B1) $-(\mathrm{B} 6)$ with the above $\rho(x)$ is

$$
\varepsilon=1, \quad \theta(x)=\arctan (\sqrt{2} x),
$$

which can be verified easily by substituting it into Eqs. (B1)-(B6). The following expressions, obtained from (B13), are helpful:

$\theta^{\prime}=\sqrt{2} /\left(1+2 x^{2}\right), \quad \theta^{\prime \prime}=-2^{5 / 2} x /\left(1+2 x^{2}\right)^{2}$,

$\sin (2 \theta)=2 \tan \theta /\left(1+\tan ^{2} \theta\right)=2^{3 / 2} x /\left(1+2 x^{2}\right)$,

$\sin ^{2} \theta=\tan ^{2} \theta /\left(1+\tan ^{2} \theta\right)=2 x^{2} /\left(1+2 x^{2}\right)$.

After substituting solution (B13) [with (B14)] into (B8) and (B10), we get

$$
\begin{aligned}
& t_{s}(x)=\frac{1}{8} \frac{\left(\rho^{\prime}\right)^{2}}{\rho}-\frac{1}{4} \rho^{\prime \prime}+\frac{\rho}{\left(1+2 x^{2}\right)^{2}} \\
& \frac{\delta T_{s}}{\delta \rho(x)}=\frac{1}{8}\left[\frac{\rho^{\prime}}{\rho}\right]^{2}-\frac{1}{4} \frac{\rho^{\prime \prime}}{\rho}+\frac{1-2 x^{2}-4 x^{4}}{\left(1+2 x^{2}\right)^{2}} .
\end{aligned}
$$

The last expression, with the help of $\rho(x)$ in the form of (B11), reduces to

$$
\begin{aligned}
\frac{\delta T_{s}}{\delta \rho(x)}=[ & \frac{1}{2}\left(-1+7 x^{2}+8 x^{4}-4 x^{6}\right) \\
& \left.+\left(1-2 x^{2}-4 x^{4}\right)\right] /\left(1+2 x^{2}\right)^{2}=\frac{1}{2}-\frac{1}{2} x^{2},
\end{aligned}
$$

i.e., negative $\mathrm{HO}$ potential shifted by a constant, in agreement with the basic Euler-Lagrange equation of densityfunctional theory. Constraints (B4) and (B5) make the solution unique (to irrelevant possible trivial factor -1 ).

At this point of discussion $t_{s}(x)$, Eq. (B8), seems to be a nonlocal functional of $\rho(x)$, because knowledge of $\rho(x)$ in a whole, infinite domain is necessary to solve Eqs. (B1)-(B6) and obtain $\theta(x)=\theta_{s}(x)$. However, in the Appendix $C$ it will be shown that, owing to a particular properties of $\mathrm{HO}$ potential, the functional dependence of $t_{s}$ on $\rho$ has a local character in this case.

Here we would like to mention a very similar problem in two-dimensional (2D) semiconductor physics, the socalled quantum dot structure [11,12]. A 2D electron gas confined by an effective potential $v_{\text {eff }}(\mathbf{r})=m \Omega^{2} r^{2} / 2$ (where $r^{2}=x^{2}+y^{2}, m$ is the effective mass of $2 \mathrm{D}$ electrons) is considered. The system still possesses symmetry around the $z$ axis. With an applied magnetic field along the $z$ direction, the total effective one-dimensional Hamiltonian can be written as

$$
\begin{aligned}
H= & \frac{1}{2 m}\left\{\frac{1}{r} \frac{\partial}{\partial r}\left[r \frac{\partial}{\partial r}\right]-\frac{l^{2}}{r^{2}}\right\} \\
& +\frac{1}{2} m \Omega^{2} r^{2}-\frac{e B l}{2 m}-\frac{e^{2} B^{2} r^{2}}{8 m},
\end{aligned}
$$


where $B$ is the strength of the magnetic field and $l$ is the angular-momentum quantum number. If only the two lowest levels are populated [i.e., $(n, l)=(0,0)$ and $(0,-1)$ states], we can obtain the following equation:

$$
\begin{aligned}
-\psi_{2} \frac{1}{r} \frac{\partial}{\partial r} & {\left[r \frac{\partial}{\partial r}\right] \psi_{1}+\psi_{1} \frac{1}{r} \frac{\partial}{\partial r}\left[r \frac{\partial}{\partial r}\right] \psi_{2} } \\
& -\frac{\psi_{1} \psi_{2}}{r^{2}}+2 m\left[\varepsilon_{2}-\varepsilon_{1}-\frac{e B}{2 m}\right] \psi_{1} \psi_{2}=0 .
\end{aligned}
$$

By introducing $\phi_{i}=\sqrt{r} \psi_{i}$ and using $\sqrt{m}\left[\varepsilon_{2}\right.$ $\left.-\varepsilon_{1}-(e B / 2 m)\right]^{1 / 2}$ as the unit of $r$, then expressing $\phi_{i}$ in terms of $\rho$ and $\theta$ according to Eq. (3.2), we obtain

$$
\theta^{\prime \prime}+\frac{\rho^{\prime}}{\rho} \theta^{\prime}+\sin (2 \theta)\left[2-\frac{1}{r^{2}}\right]=0 \text {. }
$$

This particular equation is very similar to the general $\rho-\theta$ equation (B1). The extra term $\left(\sim r^{-2}\right)$ comes from the angular momentum because this $1 \mathrm{D}$ equation is deduced from a $2 \mathrm{D}$ equation. By using an input density [e.g., $\left.\rho=2 r\left(1+r^{2}\right) \exp \left(-r^{2}\right)\right]$, one can proceed further to study the properties of a quantum dot structure (e.g., $E_{\mathrm{GS}}^{\mathrm{HF}}, T_{s}$, etc.) using the method here developed in the main text. The detailed investigation of the application to solid-state problems will be presented separately.

\section{APPENDIX C: LOCAL DEPENDENCE OF THE KINETIC-ENERGY DENSITY ON THE DENSITY OF TWO PARTICLES BOUND BY THE HARMONIC-OSCILLATOR POTENTIAL}

In a recent series of papers - an initial one by Shao and Stezowski [7], followed by a comment from Chen and Stott [9] and a reply from the former authors [10]-a question was discussed as to whether the kinetic-energy density functional $t_{s}[\rho ; x]$ can be found in an explicit form in the case of a one-dimensional two-particle system having a simple shape-invariant potential [7]. This question, narrowed further to the case of the harmonicoscillator potential, was finally answered negatively [10]: "one cannot find a closed form of kinetic-energy density," where [9] "by closed form we mean that $t_{s}$ can be expressed algebraically in terms of $\rho$ and its derivatives." In the Appendix B we showed that $t_{s}$ can be found from known $\rho$, by applying methods valid for $\rho$ generated by an arbitrary potential. Below we are going to demonstrate that, contrary to Shao's and Stezowski's conclusion [10], a positive answer to their question can be obtained, using equations derived by them which incorporate particular properties of the HO potential.

Although they use also Eq. (B1) as an important starting point, it is not treated as an eigenequation, but rather as a differential equation relating an unknown constant $\varepsilon$ and an unknown function $\theta=\theta_{s}(x)$ with the known function $\rho(x)$. In their approach $\varepsilon$ means the energy difference between the higher and lower orbits. This constant also occurs in their second starting point, Eq. (1) of [10]:

$$
\phi_{2}(x)=-(2 / \varepsilon)^{1 / 2} \phi_{1}^{\prime}(x),
$$

where $\phi_{1}$ and $\phi_{2}$ are the wave functions of the aforementioned orbits, while $\rho(x)$ is constructed out of them as $\rho=\phi_{1}^{2}+\phi_{2}^{2}$. Equation (C1) was derived in Refs. [7], [9], and [10] from the properties of the HO potential treated as the shape-invariant potential. From these two starting points, after lengthy algebra which also included integration of Eq. (B1) by parts and elimination of the unknown constant $\varepsilon$, Shao and Stezowski obtained an algebraic equation involving $\rho(x), \rho^{\prime}(x)$, and $\rho^{\prime \prime}(x)$ as well as the trigonometric functions of $\theta(x)$. We resume their investigations at just this point [Eq. (13) of [10]]:

$$
\begin{aligned}
0= & {\left[\frac{\rho^{\prime}}{\rho}\right]^{\prime} \frac{\cot \theta \cos ^{2} \theta}{\cos (2 \theta)}+\left[\frac{\rho^{\prime}}{\rho}\right]^{2} \frac{\cot \theta \cos ^{2} \theta}{\cos ^{3}(2 \theta)}\left\{2 \sin (2 \theta) \cos ^{2} \theta \cot \theta-\left[\cot ^{2} \theta+\sin (2 \theta) \cot \theta\right] \cos (2 \theta)\right\} } \\
& +\left(\frac{\rho^{\prime}}{\rho}\right]^{2} \frac{\cot \theta \cos ^{2} \theta}{\cos (2 \theta)}+\frac{1}{2}\left[\frac{\rho^{\prime}}{\rho}\right]^{2} \frac{\cot ^{2} \theta \sin (2 \theta)}{\cos ^{2}(2 \theta)}
\end{aligned}
$$

[here we corrected their equation by introducing a factor $\sin (2 \theta)$ omitted by them in the last term].

Then, applying trigonometric identities [such as these used in Eqs. (B15) and (B16)], we transform Eq. (C2) into a cubic algebraic equation

$$
\xi^{3}+(\beta-2) \xi^{2}+(4 \beta+1) \xi-\beta=0
$$

where the unknown function $\theta(x)$ enters via the expression

$$
\xi=\tan ^{2} \theta
$$

and the known function $\rho(x)$ via

$$
\beta=\frac{\left(\rho^{\prime} / \rho\right)^{2}}{\left(\rho^{\prime} / \rho\right)^{\prime}}=\left[\frac{\left(\rho^{\prime \prime} / \rho\right)}{\left(\rho^{\prime} / \rho\right)^{2}}-1\right]^{-1}
$$

While the existence of Eq. (C2) demonstrates that $\theta(x)$ is a local functional of $\rho$, i.e., some function of $\rho(x), \rho^{\prime}(x)$, etc., this function can be written explicitly, because Eqs. (C3) and (C4) are analytically solvable.

One immediately checks that the solution $\theta(x)$, Eq. (B13), found earlier on a different way, also satisfies Eq. (C3), by noting that [due to Eqs. (C4) and (B13)]

$$
\xi(x)=2 x^{2},
$$


and, according to Eqs. (C5) and (B12), that

$$
\beta(x)=2 x^{2}\left(1-2 x^{2}\right)^{2} /\left(1-8 x^{2}-4 x^{4}\right) .
$$

With $\xi(x)$ and $\beta(x)$ in the forms (C6) and (C7), Eq. (C3) becomes an identity.

The solutions of cubic equations are known as Cardano's formulas. For example, in the region of small $x$ (i.e., $x^{2}<0.11728$, so $0 \leq \beta<20.4$ ), only one root

$\xi=\bar{\xi}(\beta)=\frac{1}{3}(2-\beta)-(q+\sqrt{D})^{1 / 3}-(q-\sqrt{D})^{1 / 3}$,

where

$$
\begin{aligned}
& q=\frac{1}{27}\left(1+30 \beta-24 \beta^{2}+\beta^{3}\right), \quad p=-\frac{1}{9}\left(1-16 \beta+\beta^{2}\right), \\
& D=q^{2}+p^{3},
\end{aligned}
$$

is real and therefore it represents a physically acceptable solution of Eq. (C3). For the remaining $\beta(x)$ one should choose another root given by Cardano's formulas, such that the solution $\xi=\xi(\beta)$ is a real, positive, and monotonically increasing function of $x^{2}$. Next, the solution of Eq. (C4) in the form

$$
\theta=\arctan \left[\operatorname{sgn}(x) \xi^{1 / 2}(\beta(x))\right]
$$

assures $\theta(x)$ to be a monotonic function of $x$ in the whole infinite range. By substituting this $(\mathrm{C} 10)$ expression into Eq. (B8) with explicit relations $\xi=\bar{\xi}(\beta)$, e.g., Eq. (C8), and with $\beta$ being a function of $\rho(x), \rho^{\prime}(x)$, and $\rho^{\prime \prime}(x)$, Eq. (C5), the kinetic-energy density for the $N=2, D=1$ HO case is obtained as a local functional of $\rho: t_{s}=\bar{t}_{s}^{\mathrm{HO}}[\rho]$.

However, there is no sense in calculating $\delta \int d x^{\prime} \bar{t}_{s}^{\mathrm{HO}}\left[\rho ; x^{\prime}\right] / \delta \rho(x)$ by applying differentiation rules to this analytic expression. Since the functional $\bar{t}_{s}^{\mathrm{HO}}[\rho]$ was obtained using properties of the $\mathrm{HO}$ system with the density $\rho=\rho_{\mathrm{GS}}^{\mathrm{HO}}$, Eq. (B11), it cannot be valid for another system, described by the density $\rho_{\mathrm{GS}}^{\mathrm{HO}}+\delta \rho$, i.e.,

$$
t_{s}\left[\rho_{\mathrm{GS}}^{\mathrm{HO}}+\delta \rho\right] \neq \bar{t}_{s}^{\mathrm{HO}}\left[\rho_{\mathrm{GS}}^{\mathrm{HS}}+\delta \rho\right] .
$$

Therefore the above-mentioned functional derivative cannot be identified with $\delta T_{s}[\rho] / \delta \rho(x)$ at $\rho=\rho_{\mathrm{GS}}^{\mathrm{HO}}$. To obtain the last quantity, one should use the method described in Appendix B, valid for the $N=2, D=1$ system with $\rho$ generated by an arbitrary potential.
[1] J. C. Stoddart, A. M. Beattie, and N. H. March, Int. J. Quantum Chem. 4, 35 (1971); A. M. Beattie, J. C. Stoddart, and N. H. March, Proc. R. Soc. London, Ser. A 326, 97 (1971).

[2] R. W. Payne, J. Chem. Phys. 71, 490 (1979).

[3] M. Levy, Proc. Natl. Acad. Sci. U.S.A. 76, 6062 (1979).

[4] R. G. Parr and W. Yang, Density-Functional Theory of Atoms and Molecules (Oxford University Press, New York, 1989).

[5] A. Holas and N. H. March, Phys. Rev. A 44, 5521 (1991).
[6] Q. Zhao and R. G. Parr, Phys. Rev. A 46, 2337 (1992).

[7] J. Shao and J. J. Stezowski, Phys. Rev. A 42, 5767 (1990).

[8] K. A. Dawson and N. H. March, J. Chem. Phys. 81, 5850 (1984).

[9] J. Chen and M. J. Stott, Phys. Rev. A 44, 3387 (1991).

[10] J. Shao and J. J. Stezowski, Phys. Rev. A 44, 3389 (1991).

[11] T. Demal, D. Heitmann, P. Grambow, and K. Ploog, Phys. Rev. Lett. 64, 788 (1990).

[12] P. A. Maksym and T. Chakraborty, Phys. Rev. Lett. 65, 108 (1990). 